\title{
Tree Based Energy and Congestion Aware Routing Protocol for Wireless Sensor Networks
}

\author{
Amir Hossein Mohajerzadeh, Mohammad Hossien Yaghmaee \\ Department of Computer Engineering, Ferdowsi University of Mashhad, Mashhad, Iran \\ E-mail:ah.mohajerzadeh@stu-mail.um.ac.ir,hyaghmae@ferdowsi.um.ac.ir \\ Received June 15, 2009; revised October 15, 2009; accepted November 17, 2009
}

\begin{abstract}
Wireless Sensor Networks (WSNs) have inherent and unique characteristics rather than traditional networks. They have many different constraints, such as computational power, storage capacity, energy supply and etc; of course the most important issue is their energy constraint. Energy aware routing protocol is very important in WSN, but routing protocol which only considers energy has not efficient performance. Therefore considering other parameters beside energy efficiency is crucial for protocols efficiency. Depending on sensor network application, different parameters can be considered for its protocols. Congestion management can affect routing protocol performance. Congestion occurrence in network nodes leads to increasing packet loss and energy consumption. Another parameter which affects routing protocol efficiency is providing fairness in nodes energy consumption. When fairness is not considered in routing process, network will be partitioned very soon and then the network performance will be decreased. In this paper a Tree based Energy and Congestion Aware Routing Protocol (TECARP) is proposed. The proposed protocol is an energy efficient routing protocol which tries to manage congestion and to provide fairness in network. Simulation results shown in this paper imply that the TECARP has achieved its goals.
\end{abstract}

Keywords: Congestion Aware, Energy Efficiency, Routing Protocol, Fairness, Tree Based Routing, Wireless Sensor Networks

\section{Introduction}

Wireless Sensor Networks have been noticed and researched in recent years. These networks are composed of hundreds or thousands of sensor nodes which have many different types of sensors [1]. Using their sensors, nodes collect information about their environment such as light, temperature, humidity, motion and etc [2]. Sensor nodes should send their collected data to determined nodes called Sink. The sink processes data and performs appropriate actions. Many different paths exist between each node and sink. Using routing protocol, nodes determine a path for sending data to sink. Similar to traditional networks, routing protocols in wireless sensor networks consider different parameters in their routing process depended on their application.

WSNs have inherent and unique characteristics compared with traditional networks $[1,2]$. These networks have many limitations such as computing power, storage space, communication range, energy supply and etc. Nodes have limited primary energy sources and in most of applications they are not rechargeable [3], therefore energy consumption is the most important factor in routing process for wireless sensor networks. Node's energy is consumed due to using sensors, processing information and communicating with other nodes. Communications are the main element in energy consumption. Routing protocol directly affects communications volume; therefore energy aware routing protocols are very effective in decreasing energy consumption [4].

Routing protocols which only consider energy as their parameter are not efficient. In addition to energy efficiency, using other parameters makes routing protocol more efficient. For different applications, different parameters should be considered. One of the most important parameter is congestion management. Congestion occurrence leads to increasing packet loss and network energy consumption. Congestion occurs for different reasons in wireless networks; first, due to limited storage capacity in relay nodes. When a node receives packets more than its capacity, congestion will be occurred. Second, due to inherent shared wireless link, congestion 
occurred for similar reasons in wireless sensor networks. For example, when many nodes simultaneously decide to send packet using a shared medium, congestion will be occurred. Two main methods exist to manage congestion. Chen et al. [5] divided the techniques developed to address the problem of data congestion in WSN into two groups: congestion avoidance and congestion control. The former focuses on strategies to avoid congestion from happening and the latter works on removing congestion when it has occurred. In wireless sensor networks due to limitations in resources, avoiding congestion rather than controlling congestion is more reasonable. In congestion control mechanisms when the congestion is occurred, it will be controlled. This mechanism leads to consuming resources more. Generally even congestion control mechanism detect congestion earlier, more energy will be conserved. In this paper we consider congestion avoidance. In congestion avoidance mechanisms, by avoiding congestion occurrence, more energy is conserved. Different methods can be used to avoid congestion in wireless sensor networks. We use limited tree based routing. By definition many parameters about the created routing tree, congestion aware routing is achievable. For example, by limiting number of a node child, amount of traffic which forward to it, will be controllable.

One of the considerable parameters that affect the network energy consumption is providing fairness in nodes energy consumption. To provide fairness, network nodes' energy should be consumed equally. If one part of a network is used more than other parts, its energy will be dropped sooner than others then the network will be partitioned. If a network is partitioned, its energy consumption increases severely. Different routing strategies such as multi path, geographical, flat and hierarchical exist for WSNs. Each mentioned strategies have its own unique characteristics. Using different paths to send data to sink makes providing fairness more efficient.

Tree structure is used by routing protocols due to its inherent characteristics [6,7]. Regarding as in wireless sensor networks structure, in most of applications we have one sink and too many sender nodes, and tree structure is very popular. In many protocols, by limiting routing tree such as determining most number of nodes' Childs or the maximum depth of tree, limited tree structure is used in routing process.

In this paper, a hierarchical routing protocol which considers both energy and congestion as two main parameters in routing process is proposed. Our proposed protocol extends the routing approach in [8]. Simulation results show that TECARP catch its goals. TECARP consider two different traffics: high priority and low priority. Proposed protocol, uses best routes for high priority traffic and manages congestion, therefore we suggest TECARP for transmitting real time traffic. The rest of the paper is organized as follows: Section 2 summarizes related work, in Section 3, TECARP is discussed, Sec- tion 4 summarizes the simulation based evaluation of the TECARP routing protocol, and finally Section 5 concludes the paper.

\section{Related Work}

As mentioned before, energy consumption is the most important factor for routing protocols in WSNs. Different energy aware routing algorithms have been designed for wireless sensor networks. In [9] optimal energy consumption is the most important objective. Akkaya et al, propose [8] which is a well known algorithm for transmitting real time traffic in wireless sensor networks. It considers energy consumption in its routing procedure. It is a highly efficient and scalable protocol for sensor networks where the resources of each node are scarce. It is a Hierarchical routing algorithm. By determining real time data forwarding rate, it can support both real time and non real time traffics. Link cost function which is used by [8] is interesting.

Reactive Energy Decision Routing Protocol (REDRP) [10] is another routing algorithm for WSNs whose main goal is optimal energy consumption. This algorithm attempts to distribute traffic in the entire network fairly. Using this mechanism, it decreases total network energy consumption. REDRP is routing reactively, and uses residual node energy in routing procedure. It uses local information for routing, but nodes have a global ID which is unique for the entire network. This algorithm is divided into 4 steps. In the first step, the sink sends a control packet to all network nodes. The nodes estimate their distance to sink relatively by using this packet. The next step is route discovery. Routing is performed on demand in REDRP. This means that the routes are established reactively. After route establishment in route discovery step, data are forwarded to sink by using those routes. If a route is damaged, in route recovery step, it will be recovered or a new route will be established.

Different congestion control techniques have been proposed for wireless sensor networks [11,12]. In [11], CODA, an energy efficient congestion control scheme for sensor networks was proposed. CODA (Congestion Detection and Avoidance) comprises three mechanisms: 1) receiver-based congestion detection; 2) open-loop hop-by-hop backpressure; and 3) closed-loop multisource regulation. CODA detects congestion based on queue length as well as wireless channel load at intermediate nodes. Furthermore it uses explicit congestion notification approach and also an AIMD rate adjustment technique. In [13], two traffic types are considered: high priority and low priority. It selects a special area in network called conzone. The nodes placed in conzone only forward high priority traffic and other network nodes forward other traffics. Reference [13] proposes two algorithms: CAR and MCAR. CAR is a network-layer solution to provide differentiated service in congested sensor 
networks. CAR also prevents severe degradation of service to low priority data by utilizing uncongested parts of the network. MCAR is primarily a MAC-layer mechanism used in conjunction with routing to provide mobile and lightweight conzones to address sensor networks with mobile high priority data sources and/or bursty high priority traffic. MCAR Compared with CAR has a smaller overhead but degrades the performance of LP data more aggressively.

\section{Proposed Protocol}

Proposed protocol is a hierarchical routing protocol. Generally in hierarchical routing protocols, routing process is divided into two main different phases. Each phase is done independently. First, routing intra cluster; in this phase packets are routed between sensor nodes and cluster heads. Second, routing inter cluster; in this phase packets are routed between cluster heads and the sink. TECARP focuses on routing intra cluster. Routing inter cluster is considered for future studies. TECARP is composed of 3 phases: network clustering, creating routing tree and data forwarding. In network clustering phase, network nodes are partitioned into different clusters. During this phase cluster nodes information are delivered to cluster head. In creating routing tree phase, a limited routing tree will be created. During this phase a routing table is created for each of cluster nodes. In data transmission phase, packets are forwarded using relay nodes routing table. During the time, depend on network cluster status (congestion, fairness and energy) node's routing table will be updated. In the rest of this section, these phases are discussed in details.

\subsection{Network Clustering Phase}

In this phase, network nodes are partitioned into different clusters. In proposed protocol, clustering is done using clustering mechanism presented in [14]. During the clustering phase, a cluster head also should be elected for each cluster. At the end of this phase, information about all of the cluster nodes is delivered to cluster head. Each node in cluster sends its own information to the sink directly. It is important to know that, this phase is done once; therefore direct communication between cluster nodes and the cluster head is negligible.

\subsection{Creating Routing Tree Phase}

In this phase, using information delivered to cluster node in the former phase. In a routing tree structure, for every cluster node a path to its cluster head is determined. Cluster head knows position of all nodes located in its cluster, and then in first step in this phase, cluster head evaluates link cost between every two nodes located in their communication range [8]. For determining link cost, proposed protocol uses Formula (1).

$$
\begin{aligned}
\operatorname{Cost}_{i j} & =\sum_{k=0}^{3} C F_{k}=c_{0} \times\left(\text { dist }_{i j}\right)^{L} \\
& +c_{1}+c_{2}+c_{3} \times f\left(e_{i j}\right)
\end{aligned}
$$

- $\quad C F_{0}$ (Communication Cost $)=$ where $c_{0}$ is a weighting constant and the parameter $L$ depends on the environment, and typically equals to 2 . This factor reflects the cost of the wireless transmission power, which is directly proportional to the distance raised to some power $L$. The closer a node to the destination, the less its cost factor $C F_{0}$ and more attractive it is for routing.

- $\quad C F_{1}$ (Energy stock $)=$ this factor reflects the primary battery lifetime, which favors nodes with more energy. The more energy the node contains, the better it is for routing. Applicable in networks which have heterogeneous nodes.

- $\quad C F_{2}$ (Sensing-state cost) $=$ where $c_{2}$ is a constant added when the node $j$ is in a sensing state. This factor does not favor selecting sensing-enabled nodes to serve as relays. It's preferred not to overload these nodes in order to keep functioning as long as possible.

- $\quad C F_{3}$ (Error rate) $=$ where $f$ is a function of distance between nodes $i$ and $j$ and buffer size on node $j$ (i.e. dist $_{i j} /$ buffer_size). The links with high error rate will increase the cost function, thus will be avoided.

Cluster head using nodes' information, links cost and Dijkstra algorithm selects least cost route between every cluster node and cluster head. Using Dijkstra algorithm, route selected between every node and cluster head is optimum; and only one path is selected between each node and cluster head (only one optimal path is exist between each node and cluster head); therefore the set of all routes has a tree structure called routing tree. If a node uses selected least cost route for transmitting its traffic, network will consume least possible energy for its traffic. But, it is important to note here that, the least cost route is not always the best route. We discuss about providing fairness in network nodes energy consumption in Section 1. If always the path with least cost is selected to forward other nodes data, nodes which are located in mentioned path die so sooner than other nodes which located on paths with higher cost. Generally, if some parts of network die sooner than other parts, network will be partitioned. Partitioned network in comparison with normal network consumes energy more and has higher reliability. By using mechanism which provides fairness in network, network lifetime will be increased and then wireless sensor network can do its task more reliable and longer.

Wireless sensor networks are expensive networks. For decreasing costs, a network is used for more than one 
application. Each application has its own traffic, therefore in many cases, a sensor network should transmit different traffics with different priorities. The proposed protocol considers two traffic types: high priority and low priority traffic. Traffics based on their priority get network services. TECARP improves routing tree after constructing it; for each node, high priority traffic volume and the ability to forward other node data are determined. Based on mentioned parameters, most number of children for each node in routing tree to avoid congestion will be determined. A node's child is a node that selects former node as its next hop in its route to the sink. Usually a number in $[3,6]$ is determined as the most number of node's children. After determining most number of node's children, routing tree is modified such that no node remains with more than determined maximum number of children. Cluster head has sufficient information about all the cluster routes, therefore it can find number of each node's children simply. The cluster head decreases number of the children of nodes which have children more than the most number of children, using mechanism will be discussed in the next paragraph.

Cluster head evaluates all of the cluster nodes and then chooses nodes that have children more than most number of children. For all of neighbors of each selected nodes, proposed protocol determines the following two parameters.

- Least cost route between node and sink (using one of its neighbors except former neighbor as next hop).

- Number of children of new selected next hop neighbor.

Then among the children of each node, the one which has a neighbor with fewer children than the most number of children and least cost route to sink will be selected. Then the selected child is modified and in future it will be the child of its new qualified neighbor. In some situations, the child with appropriate conditions dose not exist, therefore exceptionally a node with children more than the most number of children is accepted. Here a routing tree with appropriate structure is ready to make for each cluster. But only cluster heads know the routes and routing tree, because they made it by itself and other nodes do not participate in mentioned process.

After selecting the best route and determining the number of children for every node, cluster head creates a routing table for all cluster nodes. A special record in routing table is considered for its best route to cluster head. In addition to best route, many paths which have lower costs in comparison to other paths are also selected to create routing table. For each of selected paths, a record is considered in routing table. Routing table has following fields: ID, residual energy, number of children, cost and average queue length. After constructing routing table, cluster head directly sends each node routing table to it.

In this phase a routing tree is created for each cluster. As mentioned before, maximum number of children for each node in cluster is constant (of course softly). Therefore traffic volume which enters to nodes is managed and controlled. This leads to decrease congestion in network; in other words, by using proposed mechanism which explained in this section congestion is avoided as much as possible. From another point of view, a routing table based on a routing tree is created for each node in cluster. Created routing tree has some records. The node depends on network conditions (congestion and neighbors residual energy), can use each of records to forward its data. Therefore all of routes participate in forwarding packets. Using different routes leads to use different neighbors to forward data.

\subsection{Data Transmission Phase}

At the end of the former phase, all the nodes have a routing table. As mentioned before, the proposed routing protocol considers two traffic types: high priority and low priority. The main goal of this phase is to determine next hop for each arrival packet at each node. In the rest of this section, the routing process which is done in each node when it receives packets with different priorities is discussed.

When a node receives a high priority packet, it performs following steps:

1) If special record in node routing table is active, this record will be selected. Otherwise Step 2 will be done. (The only special record in node routing table belongs to least cost route)

2) Among the records which have average queue length lower than threshold $\beta$, the record with lowest cost field value will be selected. If all the records have average queue length more than threshold $\beta$, Step 3 will be done.

3) Among the records, the one with the lowest cost field value will be selected.

After selecting the record, arrival packet will be sent to the node which is determined in record as next hop.

When a node receives a low priority packet, it performs the following steps:

1) Among the records which have average queue length lower than threshold $\beta$, the record with the biggest residual energy will be selected. If all of the records have average queue length more than threshold $\beta$, Step 2 will be done.

2) Among the records, the one with biggest residual energy will be selected.

After selecting the record, the arrival packet will be sent to the node which is determined in record as the next hop. Threshold $\beta$ numbered by multiply a number in $[0$, 1] to node queue capacity.

Nodes' routing table should be updated periodically; otherwise they can not play their role effectively. When a node residual energy becomes less than threshold $\alpha$, it broadcasts a message and informs its neighbors about its 
current condition. Neighbors receiving mentioned message, update the sender node's record in their routing table. Also the nodes should inform their neighbors about their average queue length. When a node queue length crosses threshold $\beta$ (goes up or down the $\beta$ ), it should inform its neighbors. Threshold $\alpha$ numbered by multiply a number in $[0,1]$ to primary available energy.

To keep routing table update is necessary for proposed protocol. If routing table records not be updated, routing process can not be efficient, because its information is old. In other side, keeping routing table update is costly. For keeping routing table update, routing protocol should force nodes to send their current condition to its neighbors periodically. This process is costly. Therefore, there is a trade off between routing efficiency and node's energy consumption. The short period leads to the efficient routing and high energy consumption; the long period leads to the lower energy consumption and less efficient routing. We do many experiments to determine best values for parameters $\alpha, \beta$.

\section{Performance Evaluation}

Akkaya considers almost similar parameters as our proposed protocol in [8]. Akkaya's algorithm is good relevant protocol to our protocol and well known routing protocol in wireless sensor networks. We simulate both TECARP and [8], using C++ language in UNIX operating system environment.

Most of hierarchical routing protocols are composed of two main parts. The first part is routing intra clusters and the second part is routing inter clusters. The first part's role is more important. The number of cluster nodes in simulations is considered as to be between 29 and 99. The communication range is determined Based on number of nodes in cluster. We consider almost a $40 * 40$ square for each cluster.

The main goal in TECARP is congestion avoidance. TECARP makes a routing tree by considering the most number of children for its nodes. When the number of children in a routing tree is limited, traffic volumes which enter to the node are limited too. Therefore when an event is occurred, congestion occurrence probability will be decreased. Another parameter which affects TECARP success in congestion management is the node's awareness about their neighbors average queue length. In this situation when nodes want to select the next hop for their data, they consider their neighbors average queue length as a parameter in decision making. The node with lower average queue length rather than other nodes has higher hope to be selected as next hop.

In the first graph, we evaluate the number of lost packets due to congestion for two protocols. In Figure 1(a) the number of packet loss in two protocols for different numbers of events is presented.

As is observable in Figure 1(a) the number of packet loss when network uses TECARP is less than another protocol. TECARP manages congestion; therefore the result shown in Figure 1 is obtained. As discussed before, one of the main influences of congestion occurrence in network is packet loss. Using congestion avoidance mechanism, congestion occurrence is decreased and therefore packet loss is decreased too.

In Figure 1(b) and 2(a) the number of received packets to cluster head is plotted versus the number of events. The number of nodes and events' place are different in two experiences. As shown in Figure 1(b) and 2(a), in the same conditions, number of packets received to cluster head in TECARP is always more than another protocol. TECARP manages congestion; it tries to reduce packet loss in nodes which are located in paths between nodes and the cluster head. Lower packet loss leads to more success in delivering packets to cluster head. Both Figure 1(b) and 2(a) show that TECARP is more successful than another protocol.

As mentioned in former sections, TECARP considers two types of traffic. Network nodes service traffics based on their priority. Both of the protocols try to deliver the best possible services to high priority traffic besides deliver suitable service to low priority traffic. In Figure 2(b) the number of loss packets from each type of traffic for both protocols is plotted versus the number of events. TP1 is high priority traffic and TP2 is low priority traffic.

As observable in Figure 2(b), proposed protocol deliv-
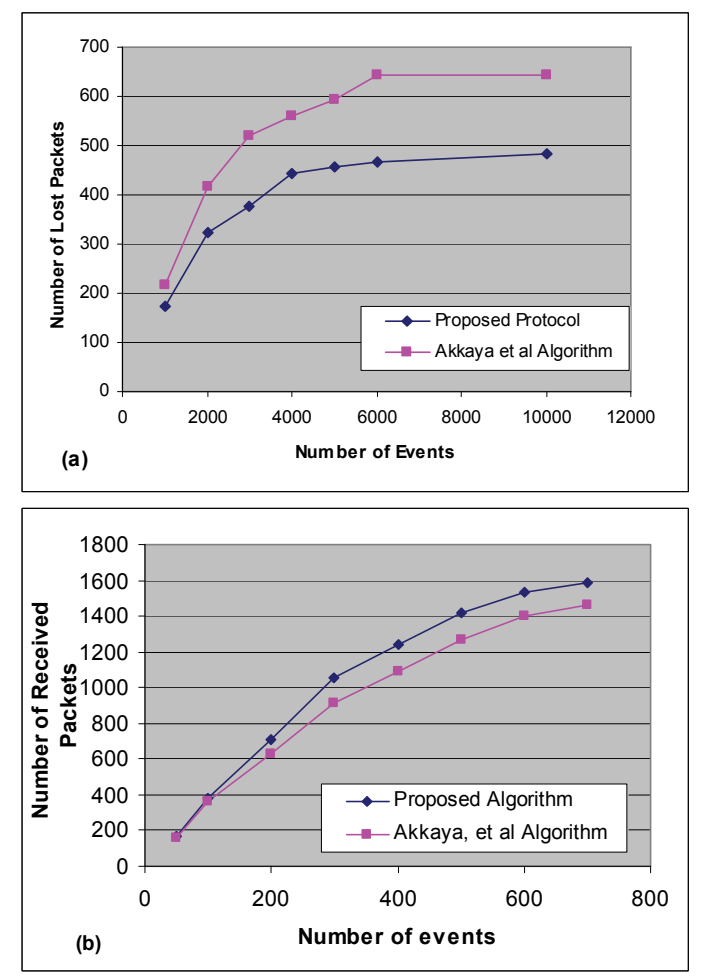

Figure 1. (a) Number of lost packets versus number of events; (b) number of received packets versus number of events. 

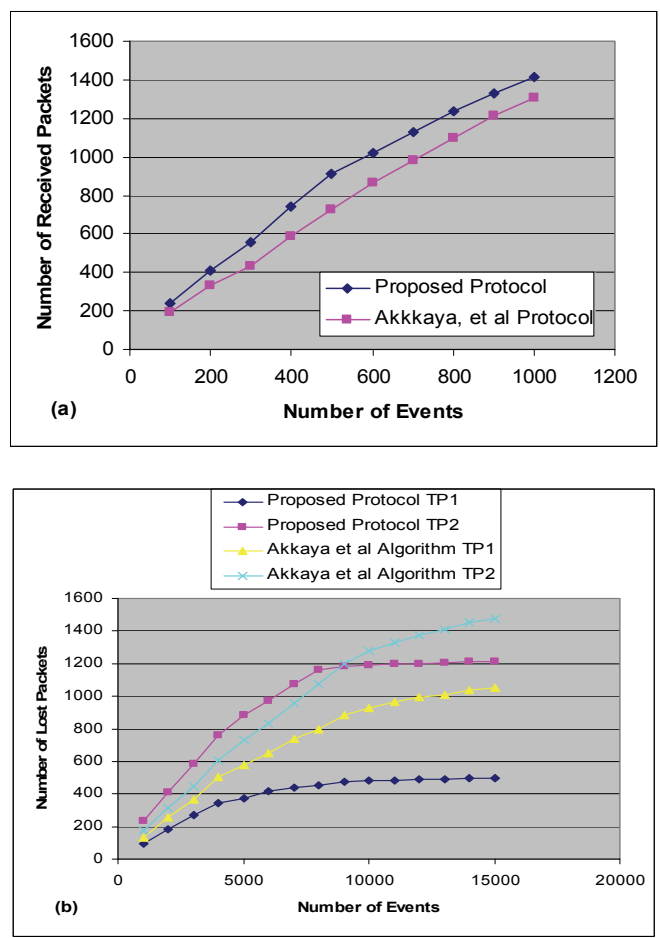

Figure 2. (a) Number of received packets to cluster head versus number of events; (b) number of lost packets versus number of events for different types of traffic.

ers better services to traffics rather than another protocol. Our proposed protocol delivers best possible services to high priority traffics. In forwarding high priority traffics proposed protocol considers only quality of delivered services to packets. But in regard of low priority traffics, in addition to quality of service, Routing protocol tries to provide fairness more efficient and to avoid congestion as much as possible. In other words, in forwarding low priority traffics proposed protocol cares more to its desirable parameters.

In Figure 3(a) performing fairness in two protocols are compared. As mentioned in Section 1, providing fairness is an important factor in wireless sensor networks routing protocols efficiency. Imagine a protocol which sends all of the data from a best cost route, in this situation, the energy of nodes located in the best route are depleted very soon; however other cluster nodes have considerable residual energy. TECARP tries to consume nodes energy as fairly as possible. Using residual energy parameter, TECARP achieves its goal. In Figure 3(a) Deviation parameter which is calculated using formula 2 is plotted versus number of events received to cluster head.

$$
\text { Deviation }=\left(\text { Node }_{i} \text { Energy }- \text { Average }\right)^{2}
$$

As shown in Figure 3(a), deviation parameter which depends on providing fairness has a better value for TECARP rather than another protocol. If a protocol provides fairness better; deviation parameter is valued less for it. For number of events less than 800, Akkaya protocol provides fairness better than our proposed protocol. Of course it is due to special routing process which is used in proposed protocol.

In Figure 3(b) a snapshot from network is plotted. In this figure, consumed energy of nodes for both of protocols is presented. As observable in Figure 3(b), proposed protocol is more successful than counterpart in providing fairness. Red columns have different heights rather than blue columns; in other words, blue columns are more equivalent in heights in comparison with red columns. The optimal condition occurs, when all of the nodes have the same residual energy.

\section{Conclusions}

In this paper, we presented a new hierarchical energy efficient routing protocol for sensor networks which considers congestion management based on paper [15]. Routing protocol divides network into many clusters, then using Dijkstra algorithm constructs a routing tree for each cluster. In routing tree, most number of children for cluster nodes is determined. Proposed protocol manages congestion, using routing tree, node's neighbors average queue length and residual energy of nodes as parameters. The effectiveness of the protocol is validated by simulation. Simulation results show that our protocol
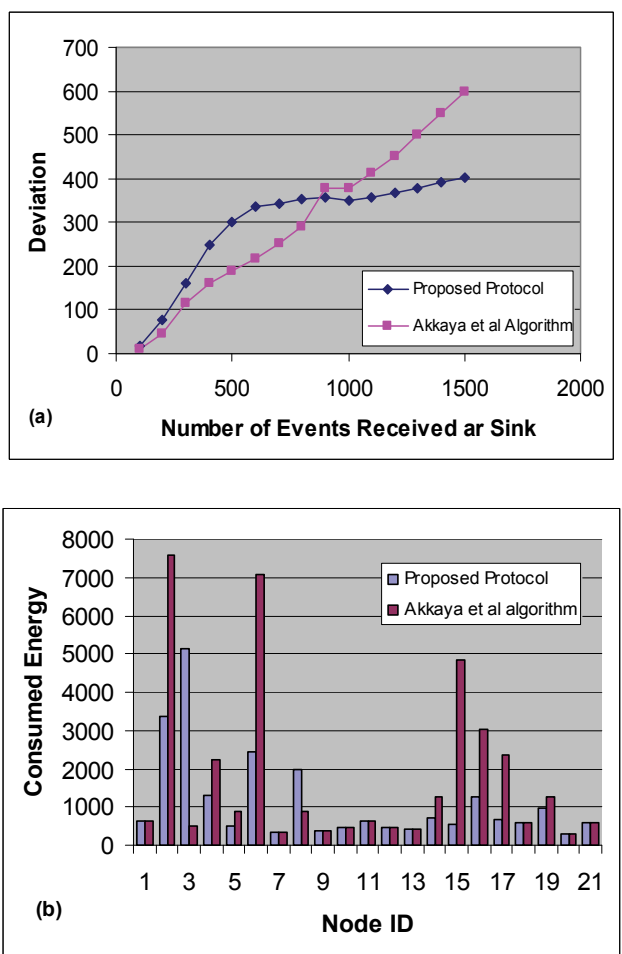

Figure 3. (a) Deviation versus number of events received at cluster head; (b) consumed energy for all cluster nodes separately. 
achieved its goals. Proposed protocol considers only intra cluster routing; we are currently extending the protocol to perform routing inter clusters.

\section{References}

[1] M. Tubaishat and S. Madria, "Sensor networks: an overview," IEEE Potentials April/May, pp. 20-23, 2003.

[2] I. F. Akyildiz, W. Su, W. Sankarasubramaniam, and E. Cayirci, "A survey on sensor networks," IEEE Communication magazine, pp. 102-114, 2002.

[3] H. Hassanein and L. Jing, "Reliable energy aware routing in wireless sensor networks," Second IEEE Workshop on Dependability and Security in Sensor Networks and Systems, DSSNS'06, pp. 54-64, 24-28 April 2006.

[4] Q. F. Jiang and Manivannan, "Routing protocols for sensor networks," 1st IEEE Consumer Communications and Networking Conference, pp. 93-98, 2004.

[5] S. G. Chen, and N. Yang, "Congestion avoidance based on lightweight buffer management in sensor networks," IEEE Transactions on Parallel and Distributed Systems, Vol. 17, No. 9, pp. 934-946, September 2006.

[6] Z. Eskandari, M. H. Yaghmaee, and A. H. Mohajerzadeh, "Energy efficient spanning tree for data aggregation in wireless sensor networks," SN'08 Workshop at ICCCN, 2008.

[7] T. Niwat, T. Yoshito, and S. Kaoru, "Tree-based data dissemination in wireless ssnsor networks," Proceedings of the IEICE General Conference (Institute of Electronics, Information and Communication Engineers), Vol. 2005, pp. S.41-S.42, 2005.
[8] K. Akkaya and M. Younis, "An energy aware QoS routing protocol for wireless sensor networks," ICDCS Workshop'03, May 2003.

[9] R. Vidhyapriya and P. T. Vanathi, "Energy aware routing for wireless sensor networks," Signal Processing, Communications and Networking, ICSCN'07, International Conference on 22-24 February 2007, pp. 545-550, 2007.

[10] Y. H. Wang, C. P. Hsu, Y. C. Lin, C. S. Kuo, and H. Y. Ho, "A routing method by reactive energy decision in wireless sensor networks," 21st International Conference on Advanced Information Networking and Applications Workshops, AINAW'07, IEEE, 2007.

[11] C. Wan, S. B. Eisenman, and A. T. Campbell, "CODA: congestion detection and avoidance in sensor networks," In Proceedings of the 1st international Conference on Embedded Networked Sensor Systems, Los Angeles, SenSys'03. ACM Press, New York, pp. 266-279, 05-07 November, 2003.

[12] M. I. Khan, W. N. Gansterer, and G. Haring, "Congestion avoidance and energy efficient routing protocol for wireless sensor networks with a mobile sink," Journal of Networks, Vol. 2, No. 6, pp. 42-49, 2007.

[13] R. Kumar, et al, "Mitigating performance degradation in congested sensor networks," IEEE Transactions on Mobile Computing, Vol. 7, No. 6, pp. 682-697, 2008.

[14] A. Abbasi and M. Younis, "A survey on clustering protocols for wireless sensor networks," Vol. 30, Issues 14-15, pp. 2826-2841, 2007.

[15] A. H. Mohajerzadeh, M. H. Yaghmaee, and Z. Eskandari, "Tree based energy efficient and congestion aware routing protocol for wireless sensor networks," IEEE ICCS, China, pp. 1707-1711, 2008. 